\title{
SISTEMA DE PUBLICIDAD POR PROXIMIDAD MEDIANTE TECNOLOGÍA BLUETOOTH COMO ESTRATEGIA PARA AUMENTAR LAS VENTAS EN LA EMPRESA LUICAM TOUCH EXCLUSIVE
}

\author{
ADVERTISING SYSTEM BY PROXIMITY THROUGH BLUETOOTH \\ TECHNOLOGY AS A STRATEGY TO INCREASE SALES IN \\ THE COMPANY LUICAM TOUCH EXCLUSIVE
}

César Bustíos Benites ${ }^{1}$ y Hugo Froilán Vega Huerta ${ }^{2}$

\begin{abstract}
RESUMEN
En este artículo se presenta la publicidad por proximidad mediante tecnología Bluetooth a dispositivos móviles y aspectos que involucran la implementación de esta estrategia como apoyo en la venta de productos y servicios en general. Se ha considerado como caso de estudio a la empresa Luicam Touch Exclusive, la cual cuenta actualmente con diversas estrategias publicitarias orientadas principalmente al apoyo de la venta de sus prendas de vestir. Se describen, detalladamente, las bondades que ofrece la tecnología Bluetooth en la vida cotidiana y en la implementación de este proyecto.
\end{abstract}

\begin{abstract}
This paper presents proximity advertising using Bluetooth technology for mobile devices and issues that imply the implementation of this strategy to support the sale of products and services in general. For this purpose, Luicam Touch Exclusive has been considered as the study case. Now, this company uses various advertising strategies aimed to support the sale of their clothing. This text describes, in detail, the advantages offered by Bluetooth technology in everyday life as well as the implementation of the project.
\end{abstract}

Keywords: Bluetooth, advertising, sales.

Palabras clave: Bluetooth, publicidad, ventas.

\footnotetext{
1 Escuela de Ingeniería Informática, Universidad Ricardo Palma. <cesarbustios@gmail.com>

2 Escuela de Ingeniería Informática, Universidad Ricardo Palma. <hugovegahuerta@hotmail.com>
} 


\section{INTRODUCCIÓN}

Con el surgimiento y la popularización de Internet, se han abierto nuevas posibilidades para el proceso de publicidad y marketing en las empresas. Por otro lado, la publicidad por proximidad que utiliza la tecnología Bluetooth abre un sinnúmero de posibilidades para convencer a los clientes de comprar los productos o servicios ofertados.

A diferencia del Marketing vía web, los clientes se encuentran a pocos metros del negocio y no sentados en una computadora portátil a kilómetros de distancia, lo que hace de esta estrategia una tendencia moderna, económica y de rápidos resultados.

En el presente artículo, se presenta una estrategia publicitaria diferente a todas las que se usan actualmente por su competencia directa. Es la misma que se implementó en la empresa Luicam Touch Exclusive, dedicada a la venta de prendas de vestir para hombres y mujeres.

\section{PRESENTACION DEL PROBLEMA}

Uno de los problemas principales de la empresa Luicam Touch Exclusive es el de saber qué cantidad de dinero debía invertir en la publicidad de los productos o servicios ofrecidos a sus clientes, es decir, qué estrategia debía usar para que fuera acertada. Sin lugar a dudas, existen numerosas estrategias disponibles en el mercado para realizar promociones o cupones de descuento que incentiven a los clientes a elegir a una empresa para satisfacer sus necesidades. Esta utiliza, actualmente, para el marketing, una página web (http://www.luicamtouch.com/).

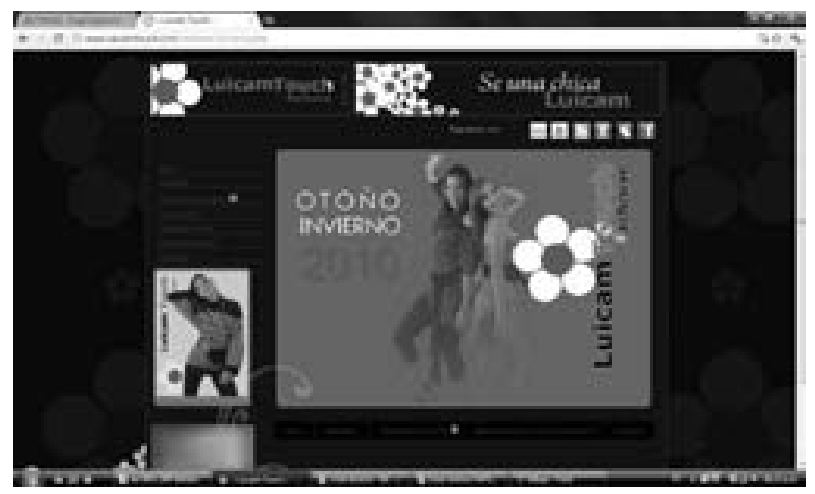

Figura 1: Página web actual de Luicam Touch.

Esta alternativa solo se limita a mostrar algunas prendas para mujeres de la gran variedad con la que cuenta la empresa. No se incluyen prendas para hombres. Esto se debe a que una página web necesita ir de la mano con otra estrategia para promocionarse. Poseen una cuenta en la red social Facebook. En ella, publican promociones y descuentos a los que también se refiere su página web. Realizan publicidad a través de flyers en determinadas ocasiones. Otra estrategia usada por la empresa es la participación en des-

files de moda que se realizan en eventos específicos y en discotecas para promocionar las marcas de diseñadores peruanos en general.

Estas estrategias dieron resultados, aunque estos no fueron los esperados, debido a una planeación deficiente. La tecnología ayudó mucho a conseguir el crecimiento de la empresa, pero el "carrito de compras" de la página web no fue, para los clientes, la mejor manera de adquirir prendas. Ellos necesitan estar presentes y probárselas frente a un espejo. Se encuentran en búsqueda de una estrategia de publicidad que no afecte demasiado el presupuesto destinado a la inversión en infraestructura o el alquiler de nuevos locales, y que permita una planeación rápida, eficiente y de fácil uso. En resumen, el problema principal de la empresa gira en torno a la falta de una planeación eficiente para llevar a cabo las estrategias publicitarias. 


\section{CONCEPTOS PREVIOS}

\section{Marketing móvil}

Se define como la utilización de medios inalámbricos interactivos para proporcionar a los clientes información personalizada sensible a la hora y al lugar. De esta manera, se promociona mercancías, servicios e ideas, lo que genera valor para todos los grupos de interés. Hay que aclarar que, cuando hablamos de medios inalámbricos o dispositivos móviles interactivos, nos estamos refiriendo no solo a teléfonos móviles, sino también a PDA (Asistentes Digitales Personales), localizadores GPS, entre otros. Sin embargo, el teléfono móvil es el más mencionado por ser el de uso más expandido. Entre las características que pueden ser aprovechadas por el marketing encontramos: ahorro, segmentación, personalización, inmediatez, alcance, interactividad, rapidez y adaptabilidad, permanencia del mensaje, y potencial efecto viral. Esto último puede entenderse como una fuente adicional de ingresos y un posible medio complementario (Steinbock, 2007).

\section{Marketing de proximidad}

El marketing de proximidad ha sabido aprovechar las nuevas tecnologías y ha encontrado en la tecnología Bluetooth y la telefonía móvil un canal de comunicación para llegar de forma directa a los usuarios y consumidores. El marketing de proximidad se basa principalmente en los dispositivos que disponen de aquella tecnología, que es utilizada para emitir información que puede ser recibida por los usuarios. Entre las ventajas más importantes de esta tecnología, cabe destacar que tanto el envío como la recepción de la información son totalmente gratuitos, aspectos que el marketing de proximidad aprovecha para realizar campañas de publicidad que representan una menor inversión en sus procesos. Otra de las ventajas importantes es la posibilidad de segmentar la información en algunos aspectos. Por ejemplo, limita su radio de acción o el tipo de información dependiendo del lugar donde se encuentre el usuario. De esta manera, un usuario con un dispositivo Bluetooth podría recibir información o publicidad que le sugiera la visita a un establecimiento, tienda, restaurante u hotel cercanos.

El marketing de proximidad se está convirtiendo en una tendencia cada vez más atractiva. La tecnología Bluetooth se aleja de la «Cultura de pago» y apuesta por la gratuidad (Arnold y Becker, 2009).

\section{Tecnología Bluetooth}

Empecemos nuestro análisis del sistema Bluetooth con un rápido vistazo de sus elementos y de su propósito. La unidad básica de un sistema Bluetooth es una piconet, que consta de un nodo maestro y hasta siete nodos esclavos activos a una distancia de 10 metros. En una misma sala (grande) pueden encontrarse varias piconets que pueden conectarse mediante un nodo puente como se muestra en la figura 2. Un conjunto de piconets interconectadas se denomina scatternet.

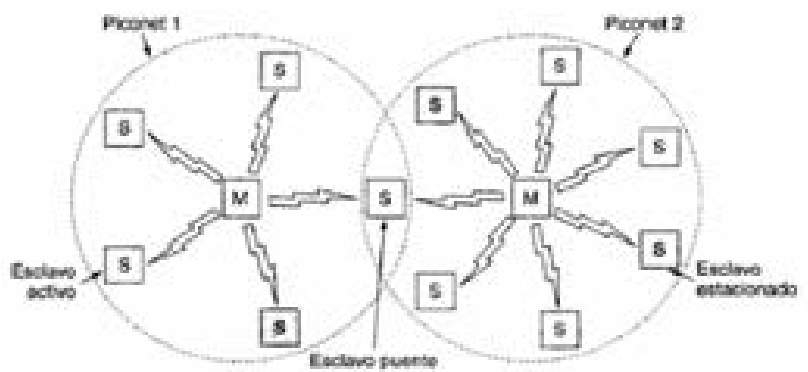

Figura 2: Dos piconets se pueden conectar para conformar una scatternet.
Además de los siete nodos esclavos activos de una piconet, puede haber hasta 255 nodos estacionados en la red. Éstos son dispositivos que el nodo maestro ha cambiado a un estado de bajo consumo de energía para reducir el desgaste innecesario de sus pilas. Lo único que un dispositivo en estado estacionado puede hacer es responder a una señal de activación por parte del maestro. 
También existen dos estados intermedios, hold y sniff, pero no nos ocuparemos de ellos en el presente texto. La razón para el diseńo maestro/esclavo es que los diseñadores pretendían facilitar la implementación de chips Bluetooth completos por debajo de 5 dólares. La consecuencia de esta decisión es que los esclavos son sumamente pasivos y realizan únicamente lo que los maestros indican. En esencia, una piconet es un sistema TDM centralizado, en el cual el maestro controla el reloj y determina qué dispositivo se comunica en un momento determinado. Todas las comunicaciones se realizan entre el maestro y el esclavo; no existe comunicación directa de esclavo a esclavo.

En la figura 3, se muestra un conjunto de aplicaciones posibles de la tecnología Bluetooth. Básicamente, se trata de la sustitución de los cables por un enlace de radio. Mediante esta tecnología, se puede acceder desde un ordenador, una cámara fotográfica digital o cualquier dispositivo electrónico a otro dispositivo Bluetooth situado en un teléfono móvil como punto de acceso a la red GSM/GPRS o UMTS. También, permite la interconexión de ordenadores creando redes adhoc. Otra de las aplicaciones es la sustitución de los cables RS-232, audio, etc., que conectan distintos dispositivos electrónicos entre sí.

Asimismo, no se debe olvidar que permite comunicaciones, incluso a través
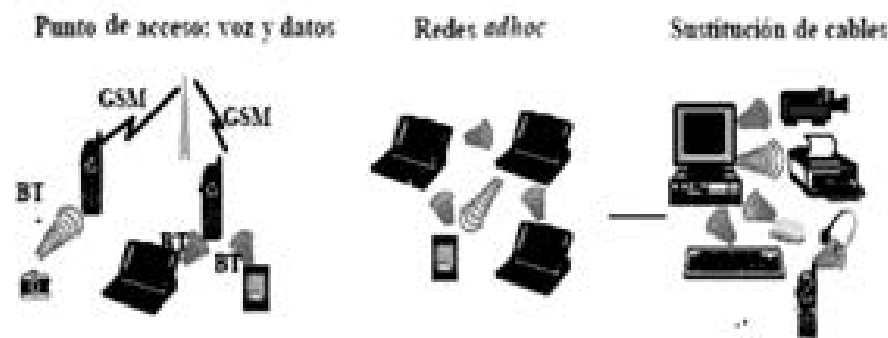

Figura 3: Aplicaciones de Bluethooth. de obstáculos, a distancias de hasta diez metros. Los promotores de Bluetooth incluyen Agere, Ericsson, IBM, Intel, Microsoft, Motorola, Nokia, Toshiba y centenares de compañías asociadas (Gehrmann et. Al., 2004).

\section{Servicio de Mensajes Cortos (SMS)}

SMS son las siglas de Servicio de Mensaje Corto. Está disponible en redes digitales GSM y permite enviar y recibir mensajes de texto de hasta 160 caracteres a teléfonos móviles vía el centro de mensajes de un operador de red (como Claro o Movistar). También, se puede enviar mensajes cortos a través de Internet, usando un sitio Web de SMS. Si el teléfono al que se envía el mensaje está apagado o fuera de cobertura, el mensaje se almacena en la red y se entrega en cuanto el teléfono se conecta de nuevo a la red (Le Bodic, 2005).

\section{Servicio de Mensajería Multimedia (MMS)}

El Servicio de Mensajería Multimedia (SMM) es la evolución de los servicios de mensaje de texto básicos a un amplio rango de contenido multimedia llevado a dispositivos móviles. Involucra tecnologías nuevas y existentes, promete servicios innovadores y es soportado por modelos de negocio probados.

\section{Banda ISM}

La banda ISM define las bandas de acceso libre regulado de 0,9 $\mathrm{GHz}$ y $5,7 \mathrm{GHz}$.

La banda de $5 \mathrm{GHz}$ está dividida en dos subbandas separadas por un vacío de entre 5,35

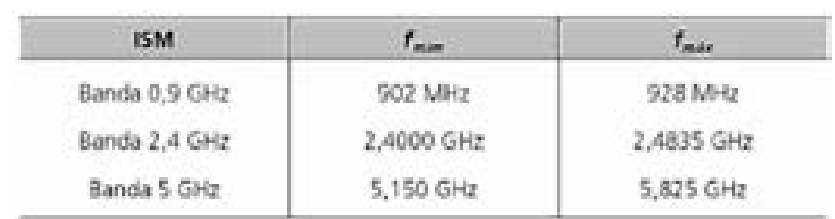

Figura 4: Acceso de banda ISM. 
GHz y 5,725 GHz. Las bandas ISM (Industrial, Scientific and Medical) son bandas reservadas internacionalmente para uso no comercial de radiofrecuencia electromagnética en las áreas industrial, científica y médica. En la actualidad, estas bandas han sido popularizadas por su uso en comunicaciones WLAN (como en Wi-Fi) o WPAN (como en Bluetooth). El uso de estas bandas de frecuencia está abierto a todos, sin necesidad de licencia, sometido únicamente a las regulaciones que limitan los niveles de potencia transmitida. Este hecho fuerza a que este tipo de comunicaciones tenga cierta tolerancia frente a errores y que utilice mecanismos de protección contra interferencias como técnicas de ensanchado de espectro. Por este motivo, las redes que funcionan en esta banda son denominadas redes de espectro ensanchado (Coleman, 2009).

\section{Protocolo OBEX}

OBEX es similar en diseńo y funcionalidad a HTTP, protocolo en el que el cliente utiliza un transporte fiable para conectarse a un servidor y recibir o proporcionar objetos. No obstante, OBEX difiere en algunos puntos importantes:

- Transporte: HTTP funciona normalmente sobre un puerto TCP/IP. OBEX, en cambio, es comúnmente implementado sobre una pila IrLAP/IrLMP/TinyTP de un dispositivo IrDA. Cuando funciona con Bluetooth, OBEX se suele implementar sobre una pila en banda Base/Link Manager/L2CAP/RFCOMM. En cualquier caso, ofrece otras posibilidades.

- Transmisiones binarias: HTTP utiliza texto legible por el ser humano, mientras que OBEX utiliza tripletes binarios llamados cabeceras (del inglés headers) para intercambiar información sobre una petición o un objeto. Estos resultan más simples de elaborar para dispositivos con características limitadas.

- Soporte para realizar sesiones: Las transacciones HTTP carecen de estado. Generalmente, un cliente HTTP establece una conexión, efectúa una sola petición, recibe respuesta y cierra la conexión. En OBEX, una sola conexión de transporte podría utilizarse para efectuar varias operaciones relacionadas entre sí. De hecho, las últimas novedades de la especificación OBEX permiten almacenar la información del estado de una conexión intacta incluso si la conexión finalizó inesperadamente (Thompson et. Al., 2008).

\section{MODELADO}

\subsection{Casos de uso del negocio (CUN)}

- Comprar productos

- Supervisar ventas

- Recibir productos

- Vender productos

- Reportar ventas diarias

- Diseñar productos

- Confeccionar productos

- Despachar productos

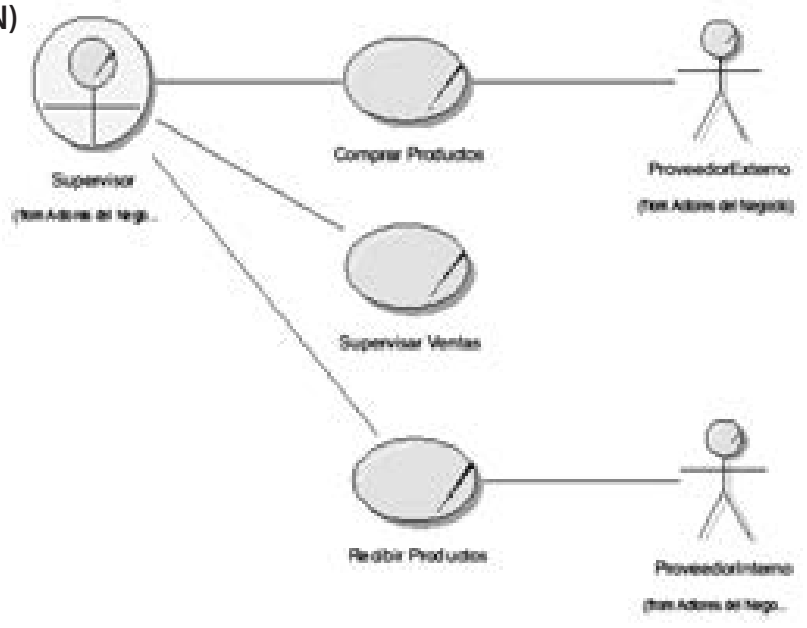




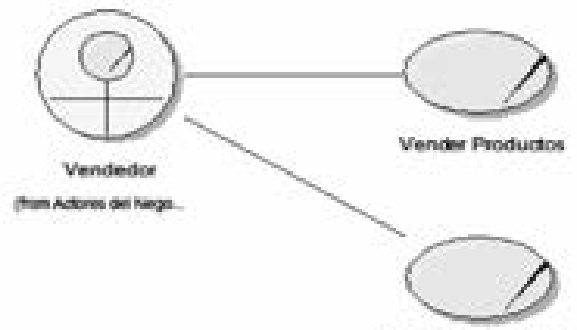

Resortar Vertas Diarias

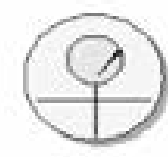

Duehedot

comaxa ainen.

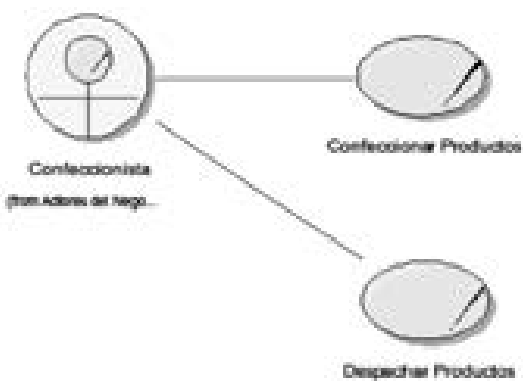

Figura 4: Diagramas de los CUN.

\subsection{Diagrama de actividades del negocio}

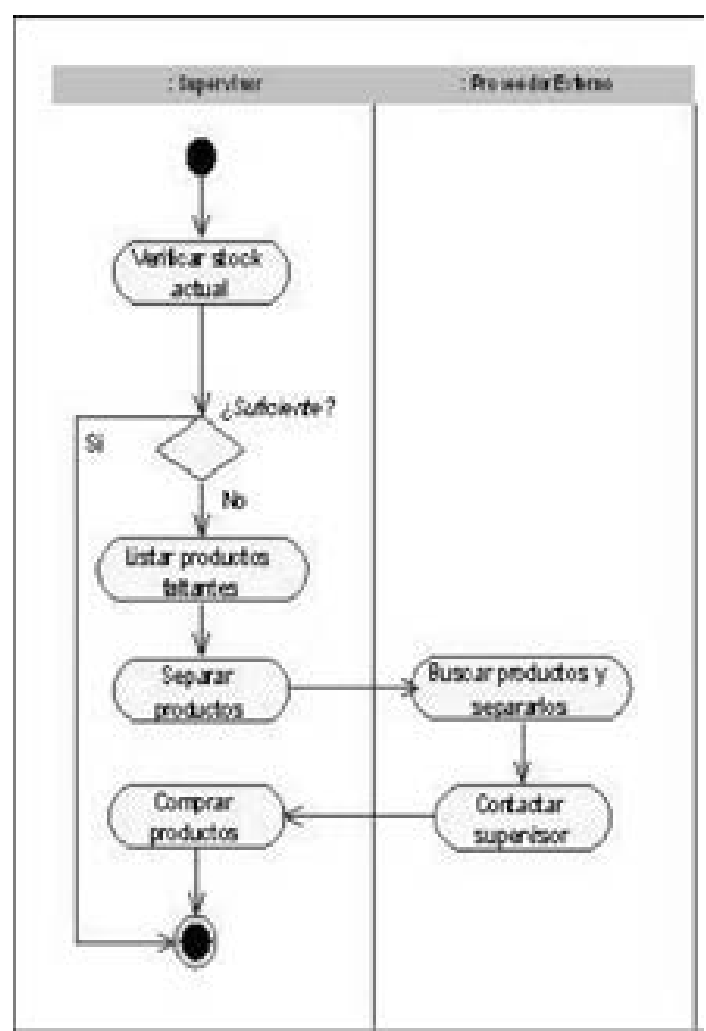

Figura 5: Comprar productos.

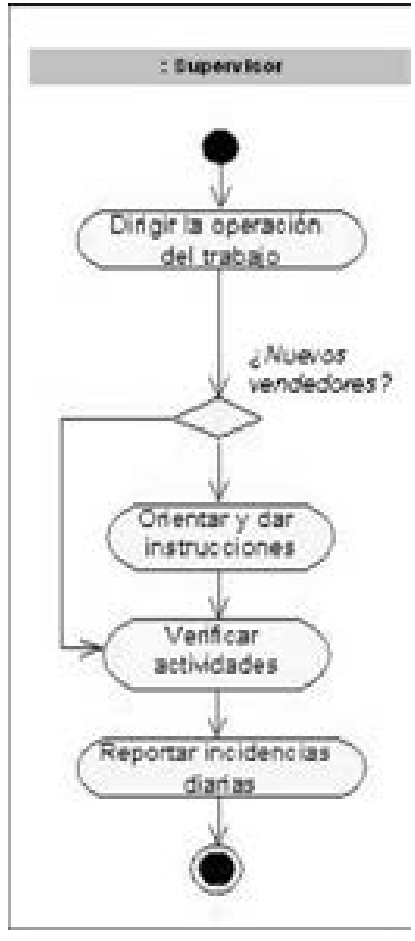

Figura 6: Supervisar ventas. 


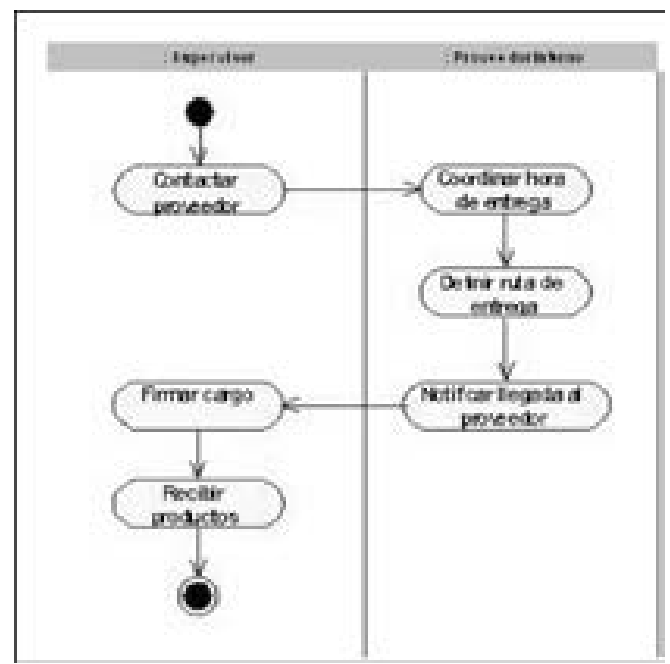

Figura 7: Vender productos.

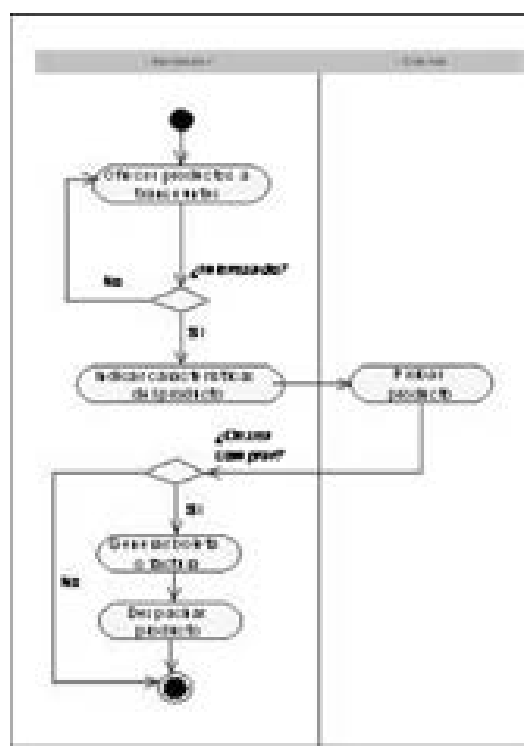

Figura 8: Recibir productos.

\subsection{Identificación de requerimientos}

A continuación se listan los requerimientos que debe cubrir el sistema en la etapa de análisis de la situación actual de la empresa Luicam Touch Exclusive.

\section{Requerimientos funcionales}

Se han identificado los siguientes requerimientos que el sistema debe satisfacer funcionalmente:

Módulo Gestión de Usuarios:

RF1: Gestionar usuarios

RF2: Gestionar usuarios

Módulo Gestión de Archivos:

RF3: Administrar archivos

RF4: Administrar archivos

RF5: Administrar archivos

Módulo Gestión de Tarjetas de Negocio:

RF6: Gestionar tarjetas de negocio

RF7: Convertir a imagen

Módulo Gestión de Campañas Publicitarias:

RF8: Administrar campañas publicitarias

RF9: Asignar cronograma

RF10: Asignar archivos

RF11: Cambiar estado

Módulo Historial:

RF12: Consultar historial

RF13: Agregar a lista negra

RF14: Exportar historial

Módulo Lista Negra:

RF15: Consultar lista negra 
RF16: Eliminar de lista negra

RF17: Exportar lista negra

Requerimientos no funcionales

RNF1: La aplicación será implementada en una PC o laptop con sistema operativo Windows XP o superior.

RNF2: Solo algunos empleados podrán usar el sistema a través de un usuario y contraseña.

RNF3: Se requiere una memoria de $256 \mathrm{Mb}$ o superior para el correcto funcionamiento del sistema así como un procesador de $1.8 \mathrm{GHz}$ o superior y un disco duro de $80 \mathrm{~GB}$ o superior.

RNF4: Es necesario que la PC o laptop tenga instalada la última versión del JRE (Java Runtime Environment), ya que el sistema será desarrollado con el lenguaje Java.

RNF5: El sistema deberá ser fiable en un 99.9\%. Un error en la aplicación puede hacer que la empresa pierda clientes potenciales y, por lo tanto, dinero.

RNF6: El sistema deberá tener un grado de escalabilidad aceptable de acuerdo al flujo de trabajo de la empresa.

RNF7: El diseño del sistema debe contemplar el uso óptimo de recursos como la conexión a la base de datos.

RNF8: El sistema deberá ser de interfaces intuitivas y fácil uso para los usuarios.

RNF9: El sistema deberá estar disponible 24 horas al día, los 7 días de la semana durante todo el año.

RNF10: Las operaciones transaccionales del sistema no deben exceder los 2 segundos.

RNF11: El código debe ser mantenido e implementado de tal forma que no obligue a generar nuevas versiones en el caso que existan cambios en los parámetros del negocio.

\subsection{Especificación de requerimientos}

A continuación, se especifican los requerimientos que fueron mencionados en la relación. Se incluyen tanto los requerimientos funcionales como los no funcionales.

\section{Requerimientos funcionales}

\section{Módulo Gestión de Usuarios:}

RF1: Gestionar usuarios:

El sistema deberá permitir la creación, eliminación y actualización de los usuarios y sus datos personales.

RF2: Gestionar usuarios:

El sistema deberá restringir el uso de diferentes funcionalidades para cada cuenta creada dependiendo del tipo de usuario (administrador o vendedor).

\section{Módulo Gestión de Archivos:}

RF3: Administrar archivos:

El sistema deberá permitir escoger diferentes archivos que serán usados posteriormente en las campañas publicitarias y ofrecer la posibilidad de eliminarlos si fuera necesario.

RF4: Administrar archivos:

Además, deberá permitir añadir un comentario cada vez que un archivo es escogido.

RF5: Administrar archivos:

Deberá mostrar datos del archivo como el nombre, el tamaño, la fecha en que fue escogido y el comentario. 


\section{Módulo Gestión de Tarjetas de Negocio:}

RF6: Gestionar tarjetas de negocio:

El sistema deberá generar automáticamente una tarjeta de negocio con los datos personales de cada usuario creado. Estas tarjetas podrán ser enviadas a través de una campaña publicitaria y eliminadas posteriormente. Considerarán campos como nombres, apellidos, teléfonos (fijo y móvil), empresa, cargo y correo electrónico.

RF7: Convertir a imagen:

También, deberá permitir la creación de una imagen, en formato .jpg, de cualquier tarjeta de contacto creada por el sistema.

Módulo Gestión de Campañas Publicitarias:

RF8: Administrar campañas publicitarias:

El sistema deberá permitir la creación, modificación y eliminación de campańas publicitarias para el envío de los archivos.

RF9: Asignar cronograma:

Además, deberá permitir establecer un cronograma para cada campaña publicitaria, es decir, el rango de fechas (inicio y fin) y el rango de horas (inicio y fin) en que estará activo.

RF10: Asignar archivos:

Asimismo, deberá permitir la asignación de los archivos a las campañas publicitarias y mostrar una lista de los archivos disponibles (incluidas las tarjetas de negocio) para ser asignados a las campañas que han sido creadas.

RF11: Cambiar estado:

De la misma manera, deberá permitir iniciar o detener una campaña publicitaria cambiando el estado de 'activo' a 'inactivo' o viceversa.

\section{Módulo Historial:}

\section{RF12: Consultar historial:}

El sistema deberá permitir visualizar el historial de los dispositivos móviles de los usuarios que fueron alcanzados por la publicidad. Mostrará el nombre del dispositivo, el archivo que recibió, el estado del envío, la campaña a la cual pertenece dicho archivo, y la fecha y hora de recepción.

NOTA: Deberá mostrar todos los dispositivos móviles de usuarios que estuvieron dentro del rango de la antena Bluetooth y que fueron alcanzados sin importar si aceptaron la publicidad.

RF13: Agregar a lista negra:

También, deberá permitir añadir cualquier dispositivo móvil a una lista negra para evitar que el sistema envíe nuevamente publicidad al dispositivo. Dicha acción requerirá un comentario.

RF14: Exportar historial:

Además, deberá ofrecer la opción de exportar el historial a un archivo externo en el formato de Microsoft Excel (.xls).

\section{Módulo Lista Negra:}

RF15: Consultar lista negra:

El sistema permitiría visualizar todos los dispositivos móviles que hayan sido agregados a esta lista con el comentario respectivo.

RF16: Eliminar de lista negra:

El sistema deberá permitir la eliminación de los dispositivos móviles que fueron agregados a la lista negra. 
RF17: Exportar lista negra:

Asimismo, deberá permitir exportar la lista negra hacia un archivo externo en formato de Microsoft Excel (.xls).

\subsection{Diagrama de actores del sistema}

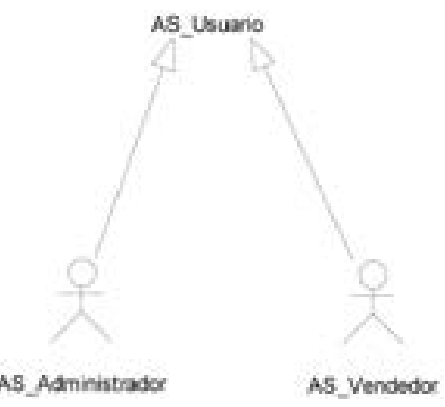

Figura 9: Diagrama de actores del sistema.

\subsection{Diagrama de paquetes}

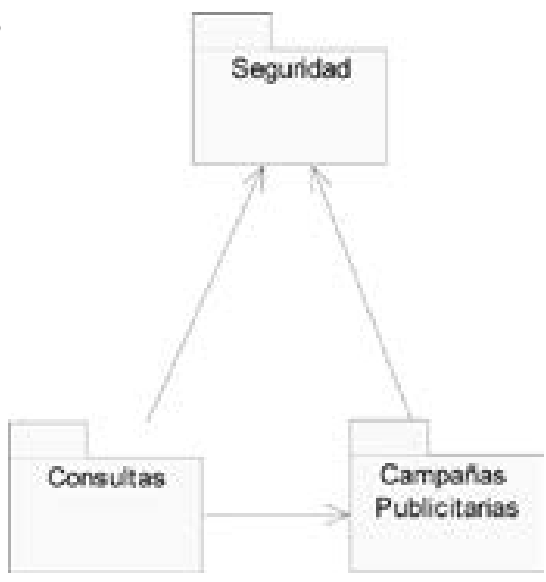

Figura 10: Diagrama de paquetes del sistema.

\subsection{Casos de uso de sistema por paquetes}

Paquete Seguridad

- Iniciar sesión

- Modificar información

- Gestionar usuarios

Paquete Consultas

- Consultar historial

- Agregar a lista negra

- Consultar lista negra

- Eliminar de lista negra

Consultas

Seguridad

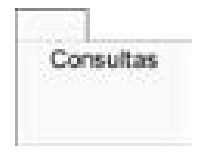


- Exportar historial

- Exportar lista negra

Paquete Campañas publicitarias

- Administrar campańas publicitarias

- Administrar archivos

- Gestionar tarjetas de negocio

- Convertir a imagen

Publicharias

- Asignar cronograma

- Cambiar estado

- Asignar archivos

\subsection{Diagrama de los casos de uso de sistema}

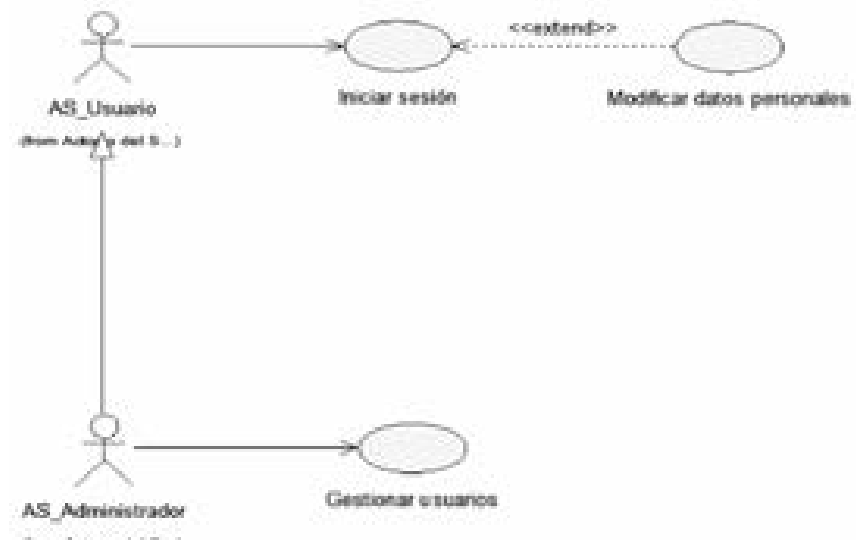

Figura 11: Usos de sistema.

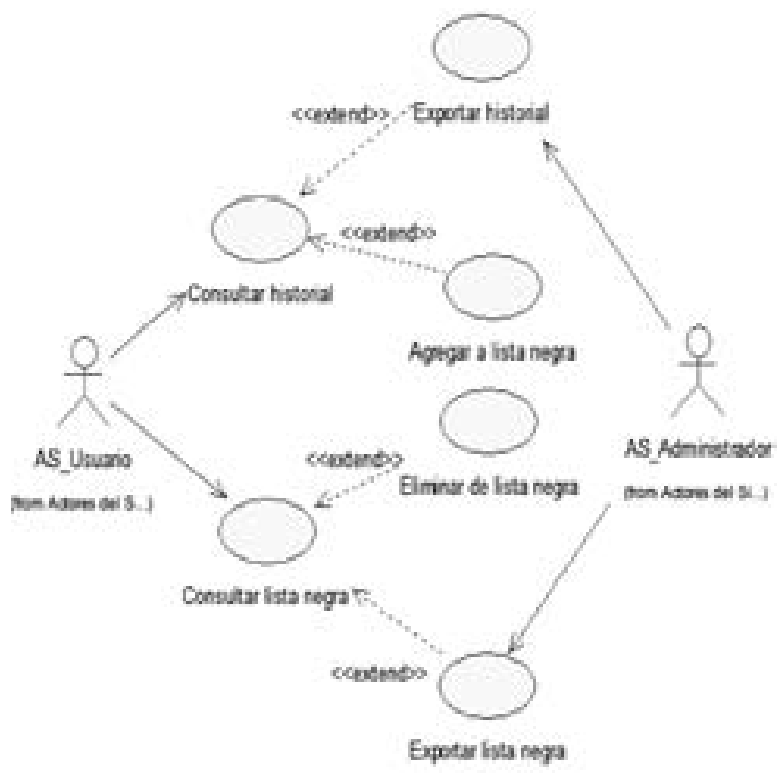

Figura 12: Diagrama de CUS del paquete consultas. 


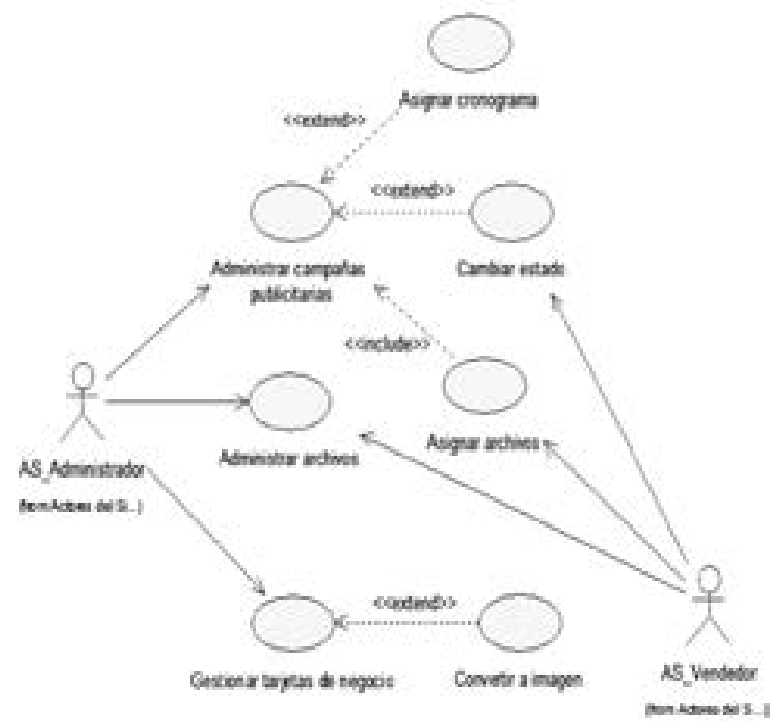

Figura 13: DCUS del paquete campañas publicitarias.

\subsection{Especificación de los CUS}

Por cuestiones de espacio, de los tres paquetes definidos (Seguridad, Consultas, Campañas Publicitarias), solamente mostraremos dos especificaciones de casos del paquete Campańas Publicitarias.

\section{ECUS Administrar campañas publicitarias}

Este caso de uso permite al usuario gestionar las campañas publicitarias, lo que incluye la creación, modificación y eliminación de campañas. Las diferentes acciones que pueden realizarse con las campañas dependen del tipo de usuario.

\section{Flujo básico}

1) Administrador: inicia sesión ingresando su nombre de usuario y contraseña.

2) Sistema: muestra la aplicación.

3) Administrador: selecciona la pestaña "Campañas Publicitarias".

4) Sistema: muestra las campañas creadas previamente (si las hubiera) y los botones "Nueva campaña", "Guardar cambios" y "Eliminar campaña"

4a) Si presiona el botón "Nueva campaña", ver subflujo "Nueva campaña”.

\section{Subflujos}

- Nueva campaña:

1) Sistema: muestra la información necesaria para crear la campaña.

2) Administrador: llena la información y presiona el botón "Crear".

3) Sistema: confirma que la campaña fue creada, muestra la lista actualizada de campańas y regresa a la pestańa "Campañas publicitarias".

- Guardar cambios:

1) Sistema: guarda los cambios realizados en la campaña publicitaria seleccionada y muestra un mensaje de confirmación. 
- Eliminar campaña:

1) Sistema: solicita que el usuario confirme la eliminación de la campaña.

2) Administrador: confirma la eliminación.

3) Sistema: elimina la campaña publicitaria seleccionada.

Flujos alternativos

En el paso 2) del subflujo "Nueva campaña”, si el administrador decide cancelar la operación:

2a) Sistema: regresa a la pestaña "Campañas publicitarias".

En el paso 1) del subflujo "Eliminar campaña", si el administrador decide cancelar la creación:

1a) Sistema: regresa a la pestaña "Campańas publicitarias".

\section{ECUS Asignar cronograma}

Esta opción permite al usuario administrador asignar un rango de fechas y horas a la campaña publicitaria si lo considera necesario.

\section{Flujo básico}

Administrador: inicia sesión ingresando su nombre de usuario y contraseńa.

2) Sistema: muestra la interfaz "Nueva campaña", en la cual el administrador puede asignar el cronograma a la campaña publicitaria. Presiona el botón "Guardar".

\section{Flujo alternativo}

En el paso 4), si el administrador decide cancelar la creación de la campańa publicitaria:

4a) Sistema: regresa a la pestaña "Tarjetas de negocio".

\subsection{Diagrama del modelo conceptual}

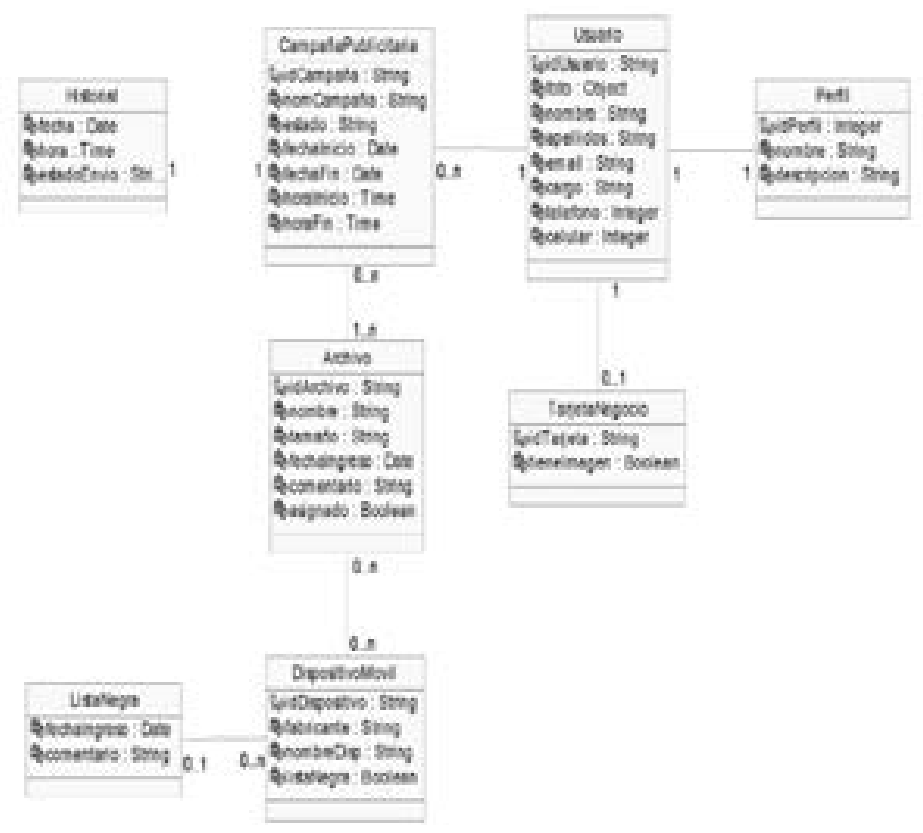

Figura 14: Diagrama del modelo conceptual. 


\subsection{Prototipos de la solución}

Por cuestiones de espacio, ahora mostraremos algunos prototipos del paquete de Seguridad y del paquete de Consultas. Por su parte, el paquete de Campañas Publicitarias lo mostraremos en forma completa.

\subsubsection{Prototipos del paquete seguridad}

CUS Modificar datos personales:

Usuario administrador/vendedor:
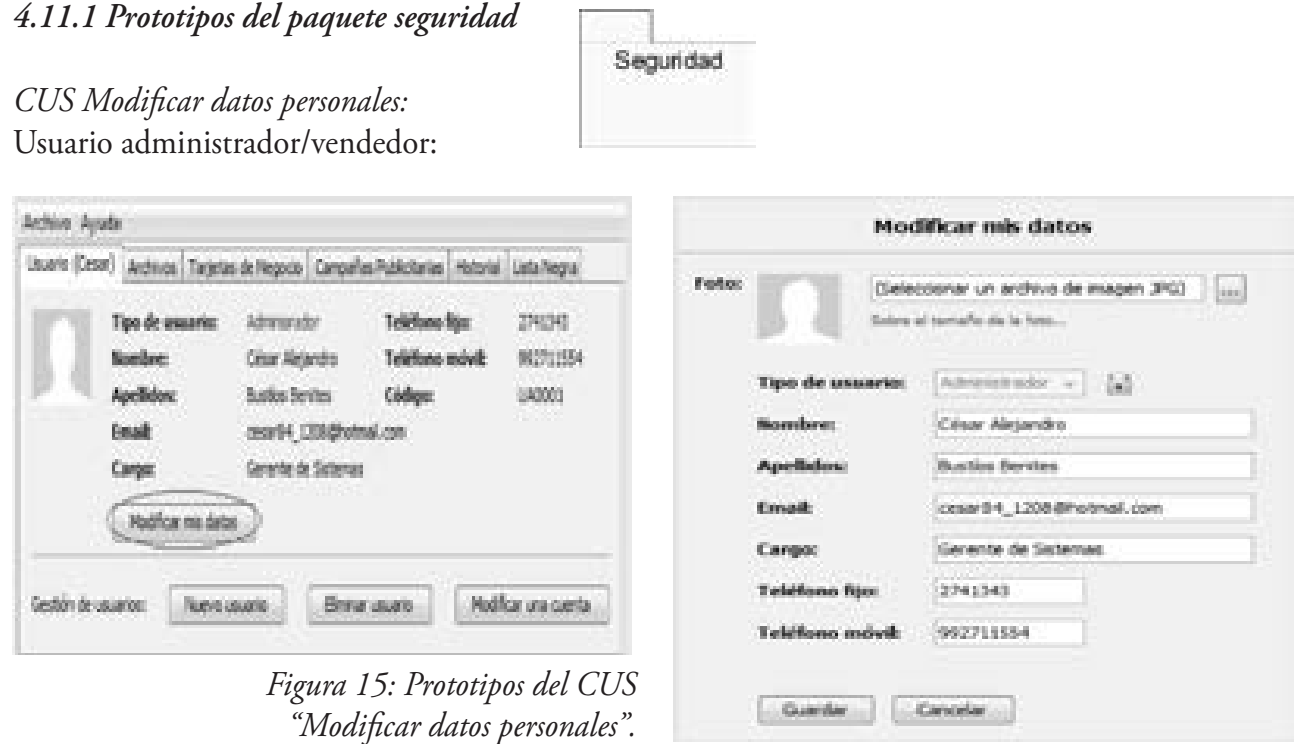

Figura 15: Prototipos del CUS "Modificar datos personales".

\subsubsection{Prototipos del paquete consultas}

CUS Consultar Historial:

Usuario administrador/vendedor:

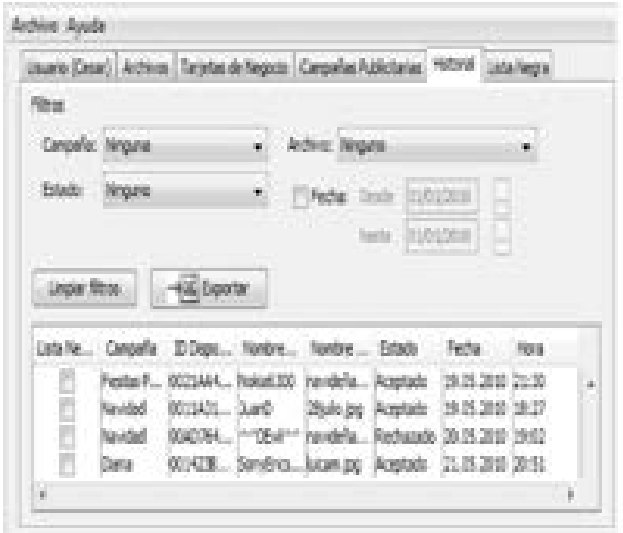

Figura 16: Prototipo del CUS "Consultar historial".
CUS Agregar a lista negra:

Usuario administrador/vendedor:

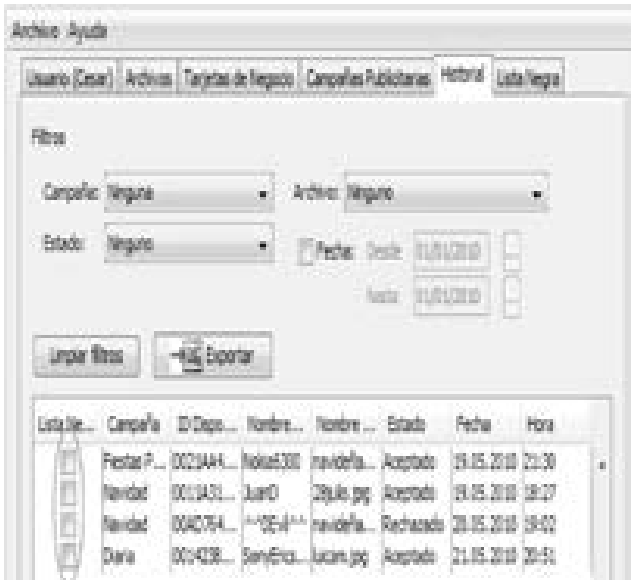

Figura 17:Prototipo 1 delCUS "Agregaralista negra". 
CUS Exportar historial:

Usuario administrador:

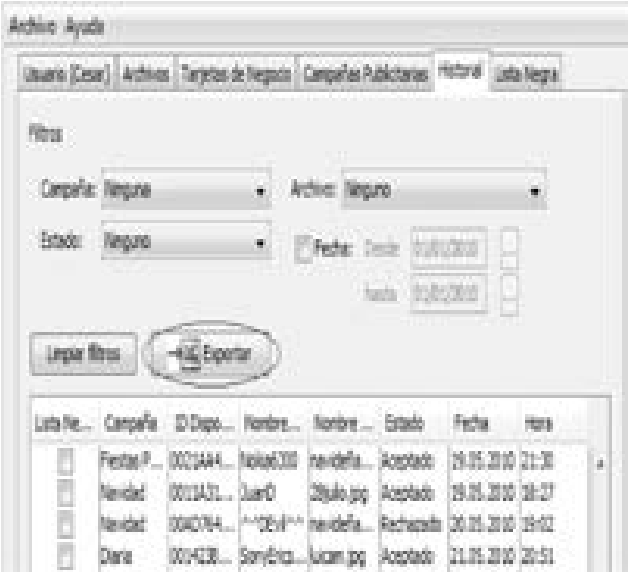

Figura 18: Prototipo del CUS "Exportar historial".
CUS Consultar lista negra:

Usuario administrador/vendedor

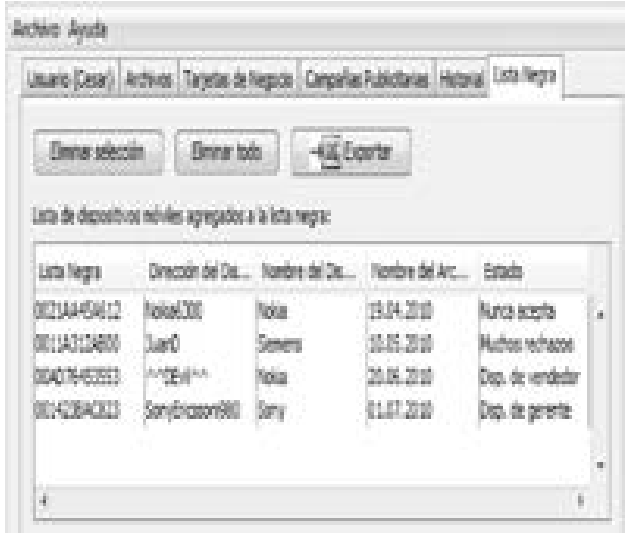

Figura 19: Prototipo del CUS "Consultar lista negra"

\subsubsection{Prototipos del paquete campañas publicitarias}

CUS Administrar campañas publicitarias:

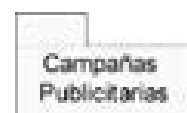

Usuario administrador:
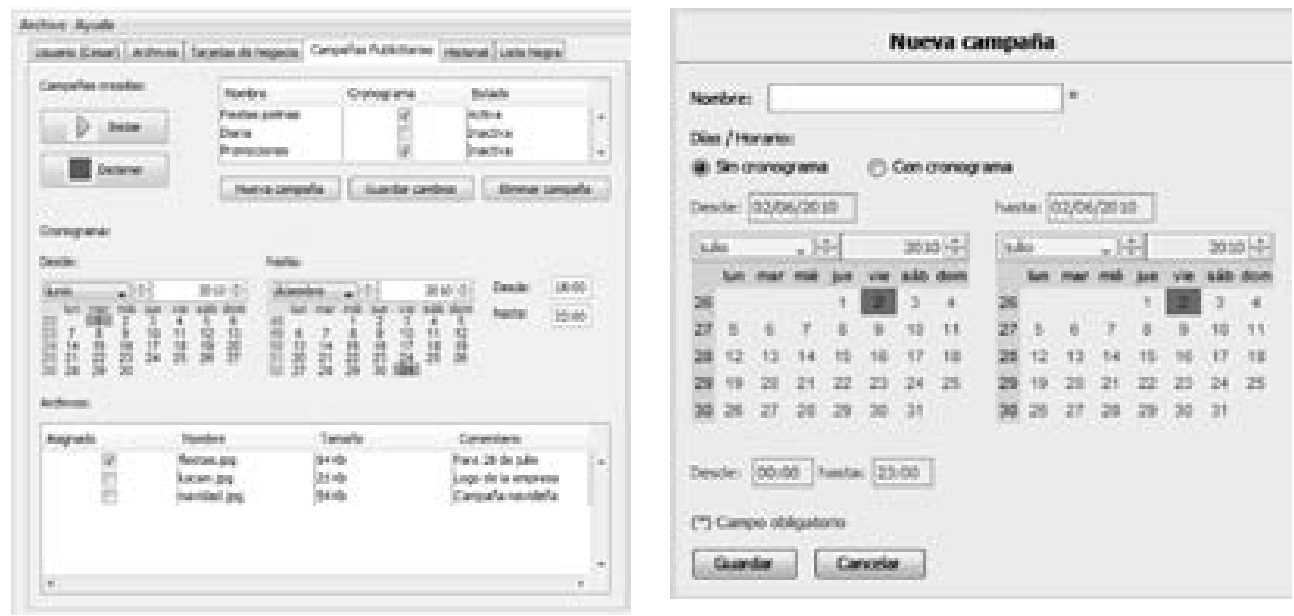

Figura 20: Prototipos del CUS "Administrar campañas publicitarias" 
CUS Asignar cronograma:

Usuario administrador:

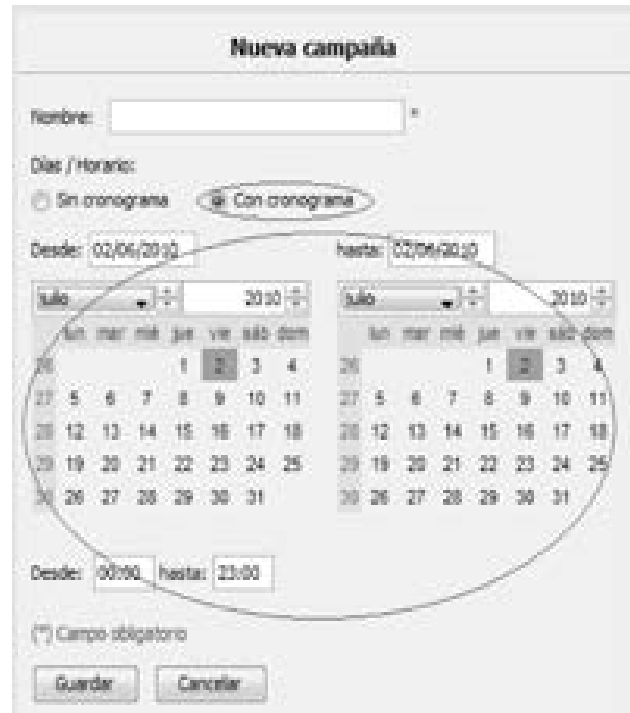

Figura 21: Prototipo del CUS "Asignar cronograma".

CUS Asignar Archivos:

Usuario Administrador:

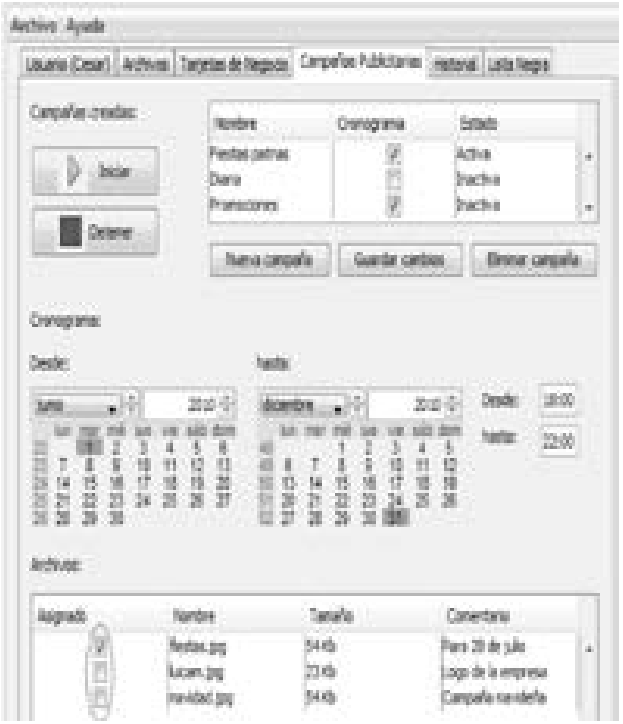

Figura 23: Prototipo del CUS "Asignar archivos"
CUS Cambiar estado:

Usuario administrador/vendedor

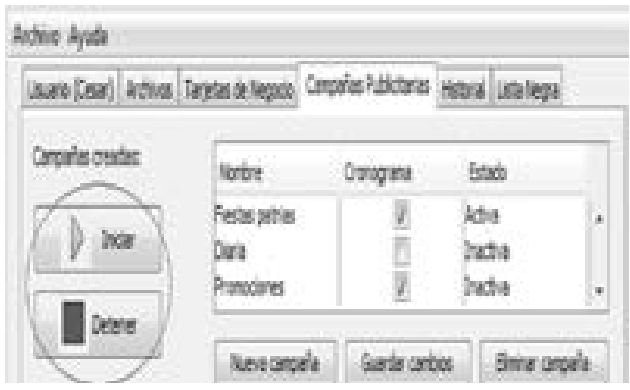

Figura 22: Prototipo del CUS "Cambiar estado".

CUS Administrar archivos:

Usuario administrador/vendedor:

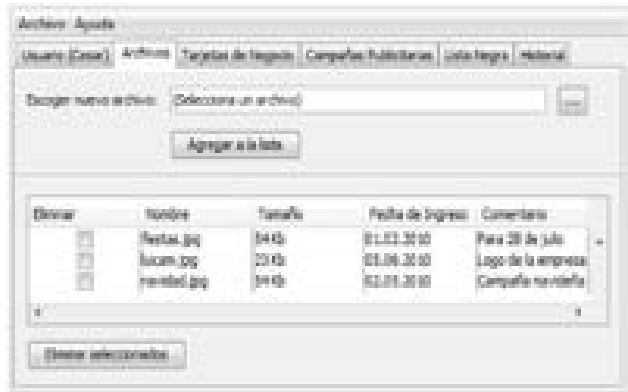

Figura 24: Prototipo del CUS "Administrar archivos"

CUS Gestionar tarjetas de negocio:

Usuario administrador:

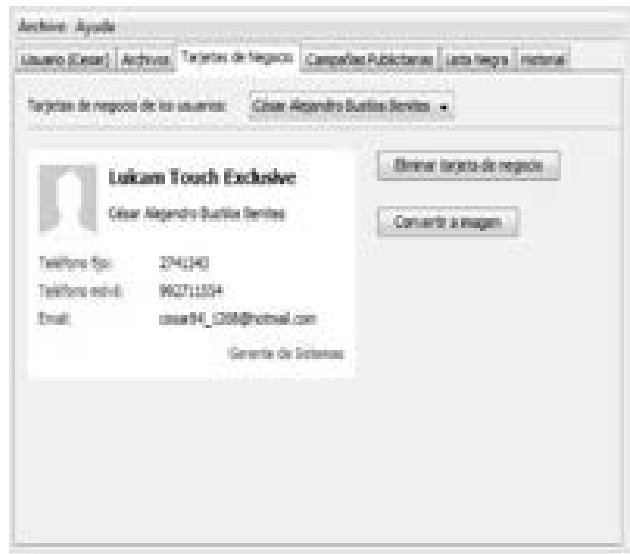

Figura 25: Prototipo del CUS "Gestionar tarjetas de negocio". 


\section{CUS Convertir a imagen:}

Usuario administrador:

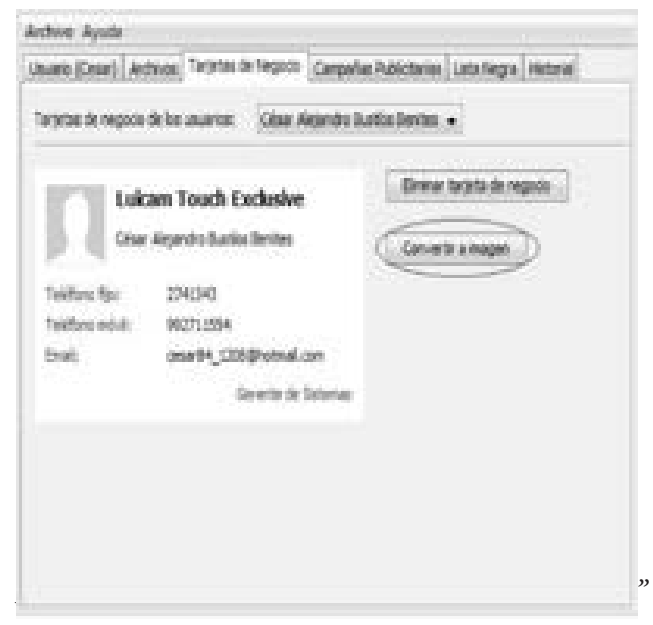

\section{RESULTADOS}

- Gracias a la implementación de este sistema, al final del año 2010, en el primer semestre del 2011 ha incrementado sus ventas en $17 \%$

- La tendencia del incremento de las ventas es creciente

- Reducción de gastos en publicidad de 50\%

- Transferencia de trabajadores dedicados al volanteo de publicidad al área de ventas.

\section{CONCLUSIONES}

- Debido al bajo costo que representa la implementación de esta solución en particular, se hace totalmente viable para cualquier empresa que desee publicitar sus productos o servicios basándose en la tecnología Bluetooth.

- Una de las ventajas principales del método escogido, basado en la gestión de campañas publicitarias, es el hecho de poder administrar la publicidad que será enviada dependiendo de la campaña que se desea utilizar y esto, a diferencia de las soluciones que no cuentan con esta característica, conlleva a un ahorro significativo de esfuerzo y tiempo.

\section{REFERENCIAS}

Arnold, J., Lurie, I., Dickinson, M., Marsten, E. y Becker, M. (2009). Web Marketing All-in-One Desk Reference for Dummies. s/l: Wiley.

Coleman, D. y Westcott, D. (2009). CWNA Certified Wireless Network Administrator Official Study Guide: Exam PW0-104. s/l: Wiley.

Fernández, E. (2004). Conocimientos y aplicaciones tecnológicas para la dirección comercial. s/l: Esic Editorial. 
Gehrmann, C., Persson, J. y Smeets, B. (2004). Bluetooth Security. s/l: Artech House Le Bodic, G. (2005). Mobile messaging technologies and services: SMS, EMS, and MMS や. s/l: Wiley. Steinbock, D. (2007). The Mobile Revolution: The Making of Mobile Services Worldwide. s/l: MPG Books.

Thompson, T., Kline, P. y Bala Kumar, C. (2008). Bluetooth Application Programming with the Java APIs Essentials Edition. s/l: Morgan Kaufmann. 